\section{Size, convexity, and curvature magnitude estimations of curvilinear forms $* \dagger$}

\author{
RICARDO DOBSON \\ Mary Washington College of the University of Virginia \\ Fredericksburg, Virginia 22401
}

Groups of Os made magnitude estimates of size, convexity, and curvature of patterns along a shape continuum. College Os repeated the procedure for a given physical measure in three successive sessions, but high school Os made only one set of estimates. The apparent size for the patterns was found to grow with an exponent between .700 and .755 for high school and college Os. The magnitude estimates for convexity and curvature were less reliable than the size estimates both between college and high school Os and within college Os over successive sessions. The magnitude estimates from the high school and college Os showed a faster apparent growth of curvature than of convexity.

Much of the recent form perception research is concerned with the quantitative and unambiguous definition of the form stimulus, the multidimensional nature of form as a stimulus, and the development of functional relations between physical measures of and perceptual responses to forms. Attneave and Arnoult (1956) have indicated the need for a psychophysics of form and also introduced a means of generating various classes of forms. One of these classes has been extensively studied by Brown and his associates who (Brown $\&$ Owen, 1967) factor analyzed 80 physical measures of these forms and then subsequently used the factor scores to predict perceptual responses in various scaling and discrimination tasks (Aiken \& Brown, 1969; Behrman \& Brown, 1968; Owen \& Andolsek, 1970).

Other psychophysical investigations have been less concerned with the dimensionality of forms with different shapes than with the sensitivity to modifications of forms with the same shape. Veniar (1948) showed that the difference threshold for distortion of a square was independent of suprathreshold levels of illumination and the area of the square. Weintraub and Hake (1962) obtained invariant $d^{\prime} s$ and slopes for z-transform ROC functions from rating scales with two, three, and four response categories for the detection of a square in a sequence of squares and rectangles. Other psychophysical researchers have examined the human ability to estimate the size of forms; a series of

*The computation for this report was supported by a faculty grant from Mary Washington College of the University of Virginia.

+ Part of the results in this report were presented at the Eastern Psychological Association convention, New York, 1971. as an extension of both lines of
research. Size, curvature, and $.7,1.5$, and 3.2 . convexity attributes of curvilinear forms, which were generated according to a mathematical equation, were examined by the psychophysical procedure of magnitude estimation.

\section{Method}

\section{EXPERIMENT 1}

Observers. Thirty female undergraduate students participated in the experiment. The Os reported having normal vision or wearing corrective lenses to achieve normal vision. Participation in the experiment was voluntary. No $O$ had had previous exposure to magnitude estimation procedures.

Apparatus and stimuli. The experimental patterns were presented to Os from $35-\mathrm{mm}$ slides by a Kodak 800 Carousel projector equipped with an Ektanar 12.7-cm focal length $f / 3.5$ lens. The projected image was centered on a white posterboard, which was positioned $242.57 \mathrm{~cm}$ in front of the lens and $264.80 \mathrm{~cm}$ from the $O$, who was to one side and in back of the projector lens.

The forms were generated from the
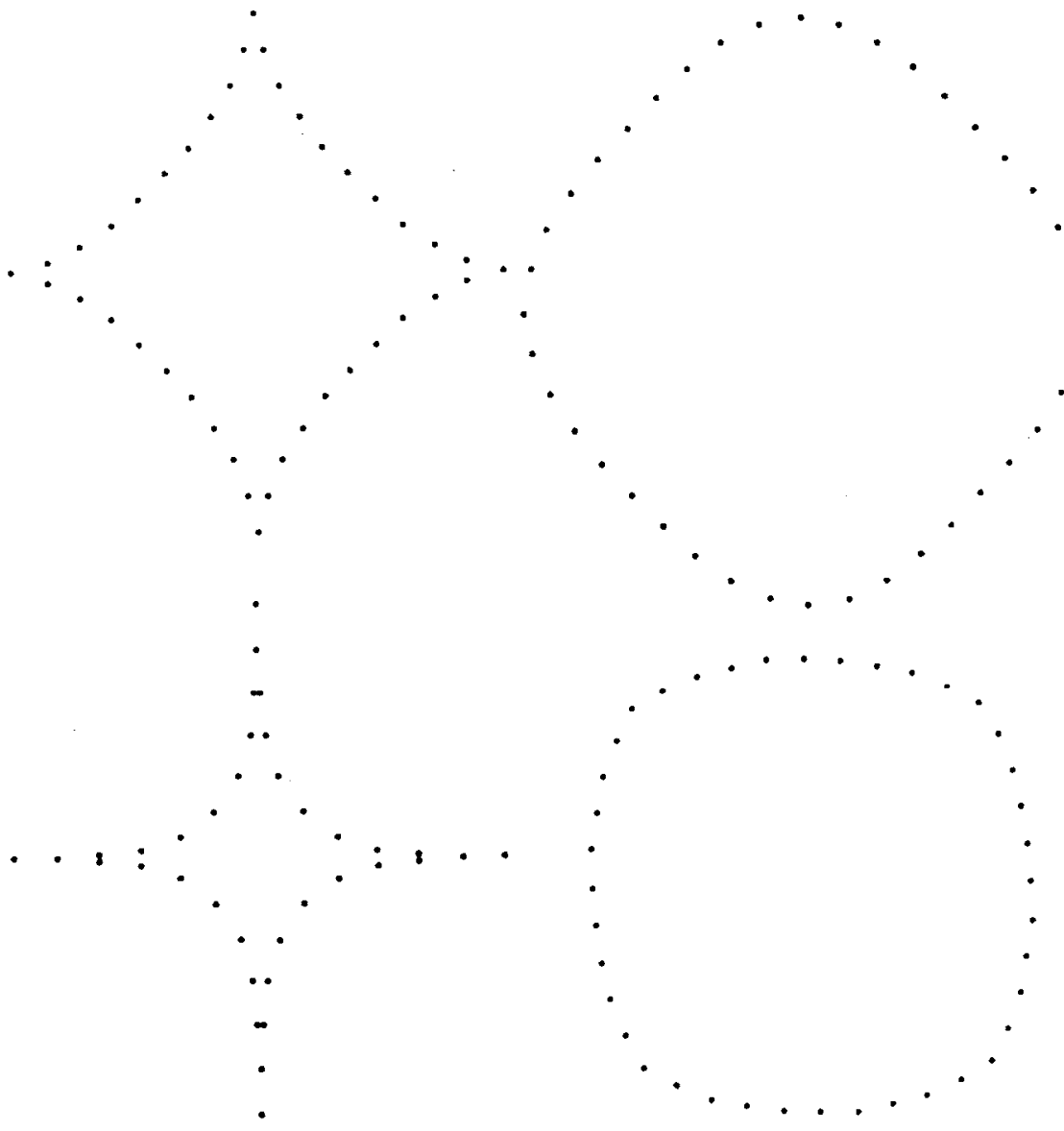

Fig. 1. A sample of four of the dotted forms which were presented to 0 s in the magnitude estimation task. The forms in the upper left, upper right, lower left, and lower right quadrants are generated when $\mathrm{n}$ of Eq. 1 is, respectively, .4, 
Table 1

Ns and Area, Convexity, or Curvature of the Patterns for Each Series

\begin{tabular}{|c|c|c|c|c|c|}
\hline \multicolumn{2}{|c|}{ Area } & \multicolumn{2}{|c|}{ Convexity } & \multicolumn{2}{|c|}{ Curvature } \\
\hline $\mathbf{N}$ & $\begin{array}{l}\text { Square } \\
\text { x Units }\end{array}$ & $\mathbf{N}$ & $\begin{array}{l}\text { Square of } \\
\text { Mean } y^{n}\end{array}$ & $\mathbf{N}$ & $\begin{array}{c}\text { Square of } \\
\text { Mean K }\end{array}$ \\
\hline .4 & .38 & 1.1 & .49 & 1.1 & .06 \\
\hline .5 & .67 & 1.2 & 1.76 & 1.2 & .20 \\
\hline .6 & .98 & 1.3 & 3.52 & 1.3 & .38 \\
\hline .7 & 1.28 & 1.4 & 5.58 & 1.4 & .54 \\
\hline .9 & 1.79 & 1.5 & 7.78 & 1.5 & .68 \\
\hline 1.3 & 2.50 & 1.8 & 14.26 & 1.6 & .79 \\
\hline 3.2 & 3.56 & 3.2 & 25.84 & 2.0 & 1.00 \\
\hline
\end{tabular}
and to expand the range of values. The
Minkowski distance function shown in Eq. 1. When Eq. 1 is plotted in two-dimensional linear coordinates,

$$
|x|^{n}+\mid y i^{n}=1
$$

the graph varies from a cross to a square. For $n$ very close to zero, it creates cross-like patterns, but as $n$ progresses toward one, a sequence of hypocycloid-like patterns are generated. When $n=1$, the graph of the equation is a square, which is rotated $45 \mathrm{deg}$ from the upright position. As $n$ increases from 1 , the sides of the square arch out, so that when $n=2$, the graph of the function is a circle. Larger values of $n$ make the form of the function approach an upright square. The patterns presented to Os had dots equally spaced along the perimeter. A sample of the experimental stimuli is presented in Fig. 1.

The forms of the Minkowski function were specified in terms of three physical measures: area, convexity, and curvature. The area for the first quadrant was computed by Simpson's rule (McCracken \& Dorn, 1964) with a step size of .05 units, and then the result was multiplied by 4 to get the area for the entire figure. The second derivative ( $\left.y^{\prime \prime}\right)$ of $\mathrm{Eq} .1$ is given in Eq. 2. The convexity of a pattern is operationally defined as the square of the arithmetic mean of the $19 \mathrm{y}^{\prime \prime} \mathrm{s}$ for values of $x$ from .05 through .95 in steps of .05 in the first quadrant.

$$
\begin{aligned}
y^{\prime \prime}= & (1-n)|x|^{2(n-1)}\left(1-|x|^{n}\right)^{(1-2 n) / n} \\
& -(n-1)|x|^{n-2}\left(1-|x|^{n}\right)^{(1-n) / n}
\end{aligned}
$$

The mean value was squared to make the convexity of the patterns positive curvature of a function at a point $(K)$ in terms of the first $\left(y^{\prime}\right)$ and second derivative is given in $\mathrm{Eq} .3$ (Graneville, Smith, \& Longley, 1957).

$$
K=\frac{y^{\prime \prime}}{\left(1+y^{\prime 2}\right)^{3 / 2}}
$$

The curvature of a pattern is operationally defined as the square of the arithmetic mean of the $19 \mathrm{Ks}$ for values of $x$ from .05 through .95 in steps of .05 in the first quadrant. The arithmetic mean of the $\mathrm{Ks}$ is squared for the same reasons that the arithmetic mean of the $y^{\prime \prime} s$ is squared. Table 1 lists the physical measurements for the three sets of seven patterns used in the experiments. Physical values of area, convexity, and curvature are given in units which specify length in $x$ units. The convexity of a pattern is a dimensionless number which denotes the rate of change of the first derivative. The curvature of a pattern has the dimensions square radians per square unit arc length.

Procedure. Thirty college students were divided into three groups of 10 Os. Each group made magnitude estimations of size, convexity, or curvature. Every $O$ performed the same magnitude estimation task in three successive sessions, which were approximately 1 week apart. The instructions were read to Os as they viewed the standard stimulus, which was always the intermediate one in a set of seven patterns. The wording of the instructions was as follows: "Call the (size, convexity, or curvature) of

\begin{tabular}{|c|c|c|c|c|c|c|}
\hline \multirow{2}{*}{$\begin{array}{l}\text { Judgment } \\
\text { Session }\end{array}$} & \multicolumn{2}{|c|}{ Size } & \multicolumn{2}{|c|}{ Convexity } & \multicolumn{2}{|c|}{ Curvature } \\
\hline & Deleted & Inverted & Deleted & Inverted & Deleted & Inverted \\
\hline 1 & $\mathbf{0}$ & 0 & 2 & 2 & 3 & 1 \\
\hline 2 & 0 & 0 & 1 & $\mathbf{3}$ & 1 & 1 \\
\hline $\mathbf{3}$ & 1 & 0 & 1 & 2 & 0 & 1 \\
\hline
\end{tabular}

Table 2

Number of $\mathrm{Os}$ Who Had Estimates Deleted or Inverted by Type of Judgment and Session of Judgment

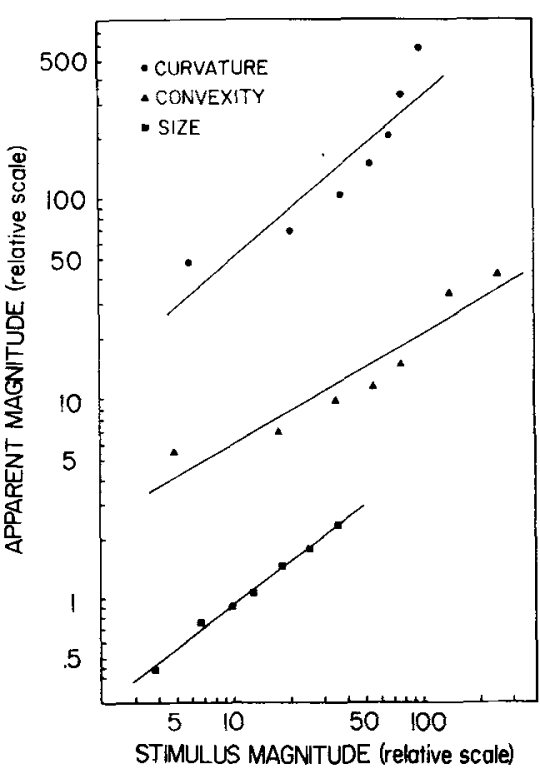

Fig. 2. The overall psychophysical functions for the size, convexity, and curvature magnitude estimations from the college Os.

this pattern 10. Your task is to estimate the (size, convexity, or curvature) of a sequence of other patterms in terms of the standard. If a subsequent stimulus pattern appears five times as (large, convex, or curved) as the standard, call it 50. On the other hand, if a subsequent pattern appears $1 / 10$ as (large, convex, or curved), call it 1 . Try not to worry about being consistent. Give whatever number you feel is appropriate for a pattern regardless of what you may have called some previous stimulus pattern. Are there any questions?"

If an $O$ asked what convexity or curvature meant, she was told to use the term as she normally did. If an $O$ asked what size meant, she was told she should estimate the apparent size of the area enclosed by the pattern. The five copies of each of the seven stimulus forms were irregularly presented so that no $O$ repeated a stimulus sequence, and no Os in the same group were exposed to an identical set of three stimulus sequences.

\section{Results}

The college Os occasionally failed to follow the instructions by not basing their estimates on the modulus, by assigning magnitude estimates which were inversely related to the physical stimulus magnitudes, or by assigning magnitude estimates which were not proportional to each other. The first difficulty may be handled by administering the procedure of modulus equalization to magnitude estimates. Modulus equalization (Lane, 
Catania, \& Stevens, 1961; Moskowitz, 1970; Panek \& Stevens, 1966) removes the variability generated by Os using different ranges of numbers without affecting the exponent of the psychophysical power function (Stevens, 1957). All magnitude estimates for groups based on type of judgment or type of judgment and session of judgment were first processed by this method before any subsequent calculations were computed.

Stevens' psychophysical power function has the form

$$
\mathbf{J}=\propto \mathbf{S}^{3} \text {. }
$$

The psychophysical function was fitted to the geometric means of the estimates of each $O$ for each session. If the fit to an $\mathrm{O}$-session record produced a negative $\beta$, the reciprocals of the estimates were used for that $\mathrm{O}$-session record in computing group data. Taking the reciprocal changes $\beta$ 's sign without altering its absolute value. If the coefficient of determination $\left(r^{2}\right)$ of the fitted function in log-log coordinates was less than .45 , then that $\mathrm{O}$-session protocol was deleted from the group analyses because $r^{2} s$ of less than .45 are not significantly different from 0 at the .05 level for 7 points (Dixon \& Massey, 1969). By this procedure, $\mathrm{O}$-session records in which the magnitude estimates were not proportional to each other were deleted. Table 2 shows the deletions and inversions by session and type of judgment.

Since the third moments were close to zero, the geometric means of the magnitude estimates were fitted by the method of least squares with Eq. 4 . Table 3 lists $r^{2}$ and the estimated values of $\alpha$ and $\beta$ based on the individual sessions for each type of judgment and for all sessions of the same type of judgment made with compatible ratio scales. For size and curvature, the value of $\beta$ remains relatively invariant over all three sessions. The last two sessions of convexity judgments have estimated $\beta$ s which are somewhat larger than .50 , but the first session has an estimated $\beta$ of only .34. The overall estimated values of $\alpha$ and $\beta$ for convexity are based on Sessions 2 and 3 because these two sessions have compatible ratio scales. The $r^{2} s$ for size estimates are uniformly greater than those for convexity and curvature. The convexity and curvature estimates are not as proportionally consistent as the size estimates.

Figure 2 shows the scatter of points about Eq. 4 for the overall data for size, convexity, and curvature judgments in log-log coordinates. The functions have been displaced along

Table 3

Estimated Parameters and Coefficients of Determination for Fit of Psychophysical Function to College Judgments

\begin{tabular}{|c|c|c|c|c|c|c|c|c|c|}
\hline \multirow[b]{2}{*}{ Session } & \multicolumn{3}{|c|}{ Size } & \multicolumn{3}{|c|}{ Convexity } & \multicolumn{3}{|c|}{ Curvature } \\
\hline & $\propto$ & $\beta$ & $\mathbf{r}^{2}$ & $\alpha$ & $\beta$ & $\mathbf{r}^{2}$ & $\propto$ & $\beta$ & $\mathbf{r}^{2}$ \\
\hline $\begin{array}{l}1 \\
2 \\
3\end{array}$ & $\begin{array}{l}9.00 \\
9.45 \\
9.50\end{array}$ & $\begin{array}{l}.74 \\
.76 \\
.70\end{array}$ & $\begin{array}{r}.98 \\
1.00 \\
1.00\end{array}$ & $\begin{array}{l}8.44 \\
4.88 \\
7.28\end{array}$ & $\begin{array}{l}.34 \\
.58 \\
.51\end{array}$ & $\begin{array}{l}.83 \\
.84 \\
.94\end{array}$ & $\begin{array}{l}37.1 \\
29.2 \\
30.8\end{array}$ & $\begin{array}{l}.86 \\
.83 \\
.77\end{array}$ & $\begin{array}{l}.86 \\
.88 \\
.88\end{array}$ \\
\hline Overall & 9.31 & .73 & .99 & $5.96^{*}$ & $.54^{*}$ & $.89 *$ & 31.8 & .81 & .87 \\
\hline
\end{tabular}

*Based on judgments from Sessions 2 and 3 only.

the $\mathrm{x}$ axis to facilitate a visual comparison of the slopes. The size estimates are more adequately described by Eq. 4 than either convexity or curvature judgments. The extreme coordinates for curvature and convexity are most poorly approximated by the psychophysical power function.

\section{EXPERIMENT 2}

The second experiment was performed with Os drawn from a different educational and age level from Experiment 1's. In this way, the generality of the Experiment 1 outcome could be investigated across $O$ populations.

\section{Method}

Observers. The Os were 24 female high school students. They reported having normal vision or wearing corrective lenses to achieve normal vision. Participation in the experiment was voluntary. No $O$ had had previous exposure to magnitude estimation procedures.

Apparatus and stimuli. The slides and position of apparatus were the same as in Experiment 1.

Procedure. The high school Os were divided into three groups of eight Os. Each group made magnitude estimations of size, convexity, or curvature. An $O$ participated in only one session, but the procedure was otherwise the same as in Experiment 1. A different stimulus sequence was administered to each $O$ within the same group.

\section{Results and Discussion}

The high school Os occasionally failed to follow the instructions in the ways that the college Os did. The size judgments had no deletions or inversions. There were three Os who had their estimates inverted for the convexity judgments, and one who had her estimates deleted from the analysis. The curvature estimates had no deletions, but one $O$ had her estimates inverted for the group analyses.

Table 4 lists $r^{2}$ and the estimated values of $\alpha$ and $\beta$ for each type of judgment made by the high school Os. The $\beta$ vaiues for convexity and curvature indicate that the high school
Os used a different ratio scale to arrive at magnitude estimates than the college Os. Both the high school and college Os had a greater $r^{2}$ for the size estimates than for the curvature and convexity estimates. The fit of Eq. 4 to the geometric means of the magnitude estimates for the high school Os is shown in Fig. 3. The size estimates are more adequately described by Eq. 4 than are the convexity or curvature estimates. While the slopes for convexity and curvature estimates are different from the college Os', the scatter about the fitted lines is very similar to that shown for the college Os in Fig. 2. The extreme points tend to be most deviant from the predicted values.

\section{GENERAL DISCUSSION}

The estimated values of $\beta$ for size in the current investigation falls within the range of values reported in the literature. Teghtsoonian (1965) found that $\beta$ might approach 1 or .76 , depending on whether the $O$ was instructed to estimate the objective or apparent size of a circle. When she instructed Os to estimate the apparent or objective size of irregular polygons, $\beta$ was approximately .8 for both forms of instruction. Stevens and Guirao (1963), employing a magnitude production procedure with squares as stimulus forms, found $\beta$ for apparent size to be .7 , but Verrillo and Graeff (1970), using a magnitude estimation procedure and filled or blanked squares as stimulus patterns, obtained a $\beta$ of .90 . In the latter report, the exact wording of the instructions was not given, but it seems that some of the Os may have judged objective size. Other investigators (Ekman, Bergström, \& Künnapas, 1956; Ekman $\&$ Junge, 1961) have found $\beta$ s for size close to .90 with circles and squares as stimulus patterns. When the $O$ cannot

Table 4

Estimated Parameters and Coefficients of Determination for Fit of Psychophysical Function to High School $O$ Judgments

\begin{tabular}{lrll}
\hline Judgment & $\alpha$ & $\beta$ & $\mathrm{r}^{2}$ \\
\hline Size & 6.19 & .71 & .99 \\
Convexity & 10.15 & .31 & .92 \\
Curvature & 18.43 & .45 & .84 \\
\hline
\end{tabular}




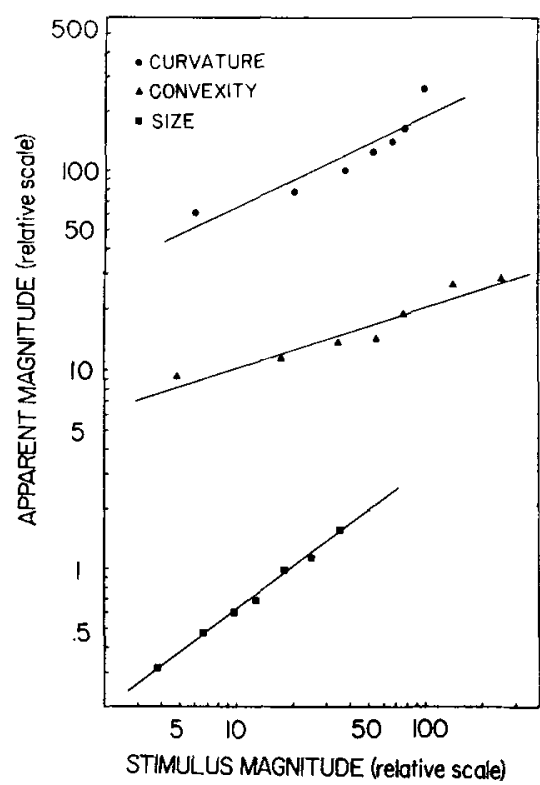

Fig. 3. The psychophysical functions for the size, convexity, and curvature magnitude estimations from the high school Os.

easily compute the relative physical area of the stimulus, for example with irregular polygons or the patterns used in the current investigation, the value of $\beta$ tends to be between .7 and .8 . When squares or circles have been used as stimulus patterns, the Os produce a wider range of outcomes. This wider range is presumably mediated by the ability of the $\mathrm{O}$ to compute the relative physical areas, and then base his estimate on either physical or perceptual size cues of the patterns.

While the psychophysical power function fitted the magnitude estimations of size without perturbations from the different patterns and $O$ groups, the same may not be said of the curvature and convexity estimates. Over the range of patterns employed, from concave to convex forms, there appears to be an orthogonal relation between the assessment of apparent size and shape. The trend of the curvature and convexity magnitude estimations is generally described by Eq. 4, but systematic deviations occurred for both high school and college Os. In addition, the exponents of the power functions were not stable between $O$ groups for either convexity or curvature estimates, and the convexity estimates from college Os did not produce consistent exponents over the three sessions.

The results suggest that it may be possible to develop psychophysical scales of curvature and convexity without fully achieving that goal. The ambiguity of the estimation task for curvature and convexity is probably greater than for size. The physical definitions of curvature and convexity are arbitrary, but future research may point to more psychophysically relevant physical measures of these stimulus attributes.

\section{REFERENCES}

AIKEN, L. S., \& BROWN, D. R. The spatial analysis of discriminability of forms in noise. Perception \& Psychophysics, 1969 5. 171-174.

ATTNEAVE, F., \& ARNOULT, M. D. The quantitative study of shape perception. Psychological Bulletin, 1965, 53. 452-471.

BEHRMAN, B. W., \& BROWN, D. R. Multidimensional scaling of form: A psychological analysis. Perception \& Psychophysics, 1968, 4, 19-25.

BROWN, D. R., \& OWEN, D. H. The metrics of visual form: Methodological dy spepsia. Psychological Bulletin, 1967, 68, 243-259.

DIXON, W. J., \& MASSEY, F. J. Instroduction to statistical analysis. New York: McGraw-Hill, 1969
EKMAN G., BERGSTRUM, B., \& KÜNNAPAS, J. A. A comparison between two psychophysical scaling methods. Report of the Psychological Laboratory, University of Stockholm, No. 37, 1956

EKMAN, G, \& JUNGE, K. Psychophysical relations in visual perception of length, area, and volume. Scandinavian Journal of Psychology, 1961, 2, 2-10.

GRANEVILLE, W. A., SMITH, P. F., \& LONGLEY, $W$. R. Elements of differential and integral calculus. New York: Ginn, 1957

LANE, H. L., CATANIA, A. C., \& STEVENS, S. S. Voice level: Autophonic scale, perceived loudness, and effects of sidetone. Journal of the Acoustical Society of America, 1961, 33, 160-167.

MCCRACKEN, D. B. \& DORN, W. S Numerical methods and Fortran programming. New Yark: Wiley, 1964.

MOSKOWITZ, $H$. R. Ratio scales of sugar sweetness. Perception \& Psychophysics, 1970, 7, 315-320.

OWEN, D. H., \& ANDOLSEK, M. A. Form recognition: Individual strategies in multivariate cue utilization. Perception \& Psychophysics, 1970, 7, 234-238.

PANEK, D. W. \& STEVENS, $S$. $S$. Saturation of red: A prothetic continuum. Perception \& Psychophysics, 1966, 1, 59-66.

STEVENS, S. S. On the psychophysical law. Psychological Review, 1957, 64, 21-37.

STEVENS, S. S., \& GUIRAO, M. Subjective scaling of length and area and the matching of length to loudness and brightness. Journal of Experimental Psychology, 1963, 66, 177-186.

TEGHTSOONIAN, $M$. The judgment of size. American Journal of Psychology, $1965,78,392-404$.

VENIAR, F. A. Difference thresholds for shape distortion of geometrical squares. Journal of Psychology, 1948, 26 . 461-476.

VERRILLO, R. J., \& GRAEFF, C. The influence of surface complexity on judgments of area. Perception \& Psy chophysics, 1970, 7, 289-290.

WEINTRAUB, D. J., \& HAKE, H. W. Visual discrimination, an interpretation in terms of detectability theory. Journal of the Optical Society of America, 1962, 52 1179-1184.

(Accepted for publication April 16, 1971.) 